\title{
MADRES POSITIVAS A Cryptosporidium parvum COMO FACTOR DE RIESGO PARA LA PRESENTACIÓN DEL PATÓGENO EN CRÍAS DE ALPACAS CON DIARREA
}

\author{
Mothers Positive to Cryptosporidium parvum As a Risk Factor for the \\ Presence of the Pathogen in Newborn Alpacas With Diarrhea
}
$\mathbf{R}^{\mathrm{a}}$ Vladimir Aguilar J. ${ }^{1}$, Armando González Z. ${ }^{2}$, Teresa López-Urbina ${ }^{1,4}$, Rosa Pera- les-Camacho ${ }^{3}$, Eloy González-Gustavson², Carlos Angulo J. ${ }^{2}$, Luis Gómez P. ${ }^{2}$

\section{Resumen}

\begin{abstract}
El objetivo del presente trabajo fue determinar si la presencia de Cryptosporidium parvum en las madres constituye un factor de riesgo para la presentación del patógeno en las crías de alpacas. Se trabajó con 698 alpacas y sus crías menores de 30 días de edad, entre enero y marzo de 2007, en las localidades de la Raya, Choquecota, Chillihua, Maranganí y Silli, provincia de Canchis, departamento del Cusco, Perú. El diagnóstico de Cryptosporidium se realizó en muestras de heces tomadas del recto y analizadas al microscopio empleando la tinción de Ziehl-Neelsen Modificado. Los análisis de riesgo se realizaron mediante una regresión logística. Se encontró que una cría con madre positiva a $C$. parvum tiene 2.1 veces más riesgo de infectarse con el parásito que una cría con madre negativa a este patógeno $(\mathrm{p}<0.05)$; asimismo, las variables sexo y presencia de diarrea no fueron significativas, en tanto que la variable lugar de crianza fue un factor de protección $(\mathrm{p}<0.009)$ para las crías pertenecientes a las comunidades en relación a las crías en granjas experimentales.
\end{abstract}

Palabras clave: alpaca, Cryptosporidium spp, Ziehl-Neelsen modificada, factor de riesgo

\section{Abstract}

The aim of the study was to establish whether the presence of Cryptosporidium parvum in the dam is a risk factor for the presentation of the pathogen in the baby alpaca. The study included 698 alpaca dams and their offspring less than 30 days of age. The sampling took place from January till March 2007 in the localities of La Raya, Choquecota, Chillihua, Maranganí and Silli in Canchis province, Cusco, Peru. Fecal samples were collected from the rectum, processed using the Modified Ziehl-Neelsen stain and observed

\footnotetext{
${ }^{1}$ Laboratorio de Microbiología y Parasitología Veterinaria, ${ }^{2}$ Laboratorio de Medicina Veterinaria Preventiva, ${ }^{3}$ Laboratorio de Histología, Embriología y Patología Veterinaria, Facultad de Medicina Veterinaria, Universidad Nacional Mayor de San Marcos, Lima

${ }^{4}$ E-mail:mtlu@terra.com.pe
} 
by microscopy. Risk analysis was done through logistic regressions. Results showed that a baby alpaca born from a positive dam to C. parvum was 2.1 times more likely to get infected with the parasite as compared with others born from a negative dam $(\mathrm{p}<0.05)$. Likewise, sex and presence of diarrhea were not significant, whereas place of breeding showed to be a protection factor for newborns in peasant communities as compared to those born in experimental station farms.

Key words: alpaca, Cryptosporidium spp, modified Ziehl-Neelsen stain, risk factor

\section{INTRODUCCIÓN}

La crianza de camélidos sudamericanos constituye una de las actividades más importantes de generación de recursos para un gran sector de la población altoandina del país. Los departamentos de Puno, Cusco y Arequipa son los mayores productores de alpacas, con el 55,15 y $10 \%$, respectivamente (FAO, 2005).

La producción de camélidos se ve seriamente afectada por diversas enfermedades de origen parasitario, bacteriano y viral. Dentro de ellas, las entidades etiológicas que participan en la presentación del síndrome diarreico neonatal de la alpaca tienen gran importancia. Entre ellas se encuentran rotavirus, coronavirus, Escherichia coli enteropatógena, Clostridium perfringens, Eimeria y Cryptosporidium parvum. En este sentido, se ha demostrado que el Cryptosporidium spp puede afectar a las alpacas jóvenes, produciendo una alta morbilidad y mortalidad, tal como ocurre en corderos y cabritos, donde la infección es más frecuente entre los 4 a 15 días de edad, produciendo cuadros diarreicos (Angus, 1990; Cármenes et al., 1993).

Diversos estudios han ayudado a encontrar el papel que tiene el Cryptosporidium en el complejo entérico neonatal de los rumiantes domésticos. En este contexto, se ha podido determinar su intervención como agente principal o secundario en las presentaciones de enfermedades diarreicas, principalmente en neonatos. El objetivo del presente trabajo fue determinar si las madres que son diagnosticadas positivas a Cryptosporidium representan un factor de riesgo para la presentación de este germen y ocurrencia de diarrea en sus crías.

\section{Materiales y Métodos}

\section{Lugar del Estudio}

Se trabajó con animales de cinco localidades de la provincia de Canchis, departamento de Cusco, Perú, ubicadas sobre los $4300 \mathrm{msnm}$. Estas fueron La Raya, Choquecota, Chillihua, Maranganí y Silli. En Chillihua se muestrearon los sectores Chiaraje y Piti, y en La Raya los sectores La Raya IVITA(UNMSM) y la Raya UNSAAC (Universidad Nacional de San Antonio de Abad). El periodo de muestreo correspondió a la temporada de parición de alpacas, entre enero y marzo de 2007. Los animales estaban en un sistema de crianza al pastoreo, alimentados con pasto natural y en algunos casos con pasto cultivado.

Las muestras se procesaron en la Estación Experimental del Centro de Investigación IVITA-Maranganí, provincia de Canchis, Cusco y se analizaron en el Laboratorio de Microbiología y Parasitología de la Facultad de Medicina Veterinaria, Universidad Nacional Mayor de San Marcos, Lima. 
Cuadro 1. Presencia de heces diarreicas y diagnóstico de Cryptosporidium parvum en crías de alpacas de la provincia de Canchis

\begin{tabular}{ccc}
\hline \multirow{2}{*}{ Diarrea } & \multicolumn{2}{c}{ Cryptosporidium $\mathrm{spp}$} \\
\cline { 2 - 3 } & Negativos & Positivos \\
\hline Ausencia & 399 & 27 \\
Presencia & 260 & 12 \\
\hline Total & 659 & 39 \\
\hline
\end{tabular}

\section{Diseño del Estudio}

Se diseñó un estudio Caso-Control, donde la presencia de Cryptosporidium en la alpaca madre se evaluó como factor de riesgo de presencia de Cryptosporidium en la cría. Se consideró que las crías con diarrea representaban casos y que las crías sin diarrea representaban controles. En este contexto, se recolectaron muestras de heces de alpacas, tanto de madres como de sus respectivas crías (hembras y machos), las cuales tenían menos de 30 días de edad. El diagnóstico de diarrea fue hecho por un Médico Veterinario y la edad fue proporcionada por el pastor de la punta o rebaño.

\section{Tamaño Muestral}

El cálculo del tamaño muestral $(\mathrm{n}=163)$ se realizó para estudios de caso control no pareado (Rothman y Greenland, 1998), con un nivel de confianza del 95\%, una potencia del $80 \%$, y una proporción esperada de exposición entre los controles de 14\% (Foroca et al., 2001). Debido a la disponibilidad de animales, se colectaron muestras fecales de 698 madres y 698 crías. Se registró la presencia de heces diarreicas tanto en la madre como en la cría, el sexo de la cría y la localidad.

\section{Manejo de las Muestras}

Las heces se recolectaron directamente del recto con el uso de bolsas plásticas rotuladas. Las heces, según su consistencia, se clasificaron en diarreicas (líquidas y pastosas) y en normales. Las muestras se fijaron, dentro de las 24 horas de su colecta, sobre láminas portaobjetos, con metanol absoluto durante 5 minutos en un vaso kopling, y luego se dejaron secar al ambiente.

Las láminas fijadas fueron teñidas empleando la tinción de Ziehl-Neelsen Modificada (Henricksen y Pohlenz, 1981). Se consideraron positivas al observar la presencia de al menos un ooquiste de Cryptosporidium. Estos se observan como organismos esféricos u ovalados de 4-6 $\mu \mathrm{m}$ de diámetro (Muñoz et al., 1993; Fayer et al., 2000), de color rojo fucsia con algunas granulaciones oscuras en su interior, que contrastan con un fondo verde (Henricksen y Pohlenz, 1981; Casemore et al., 1985).

\section{Análisis de Datos}

La presencia de Criptosporidium como factor de riesgo para la presencia de Cryptosporidium en crías de alpaca se evaluó calculando la razón de riesgos (odds ratio), empleando una regresión logística múltiple con el paquete estadístico STATA $10.0^{\circledR}$. Del mismo modo, la presencia de Crytosporidium spp en la madre y variables potencialmente confundentes como edad, sexo, raza y el lugar de crianza de las alpacas en la presencia de diarrea se evaluaron empleando una regresión logística múltiple. Las razones de riesgo se calcularon con intervalos de confianza del $95 \%$.

\section{Resultados}

El 34\% (239/698) de las madres fueron positivas a la presencia de Cryptosporidium. Asimismo, se observó diarrea en 88 madres (13\%), pero solo 8 de ellas presentaban ooquistes de Cryptosporidium en las heces.

Se observó diarrea en 272 crías (39\%), diagnosticándose Cryptosporidium en 12 de ellas; en tanto que se tuvo 27 crías sin dia- 
Cuadro 2. Diagnóstico de Cryptosporidium parvum por comunidad en alpacas de la provincia de Canchis

\begin{tabular}{lccccc}
\hline \multirow{2}{*}{ Localidad } & Total de & \multicolumn{2}{c}{ Positivas en madres } & \multicolumn{2}{c}{ Positivas en crías } \\
\cline { 3 - 6 } & muestras & $\mathrm{N}$ & $\%$ & $\mathrm{~N}$ & $\%$ \\
\hline Choquecota & 255 & 190 & 74.5 & 27 & 10.6 \\
UNSAAC (Viscanchi) & 125 & 0 & 0 & 3 & 2.4 \\
UNSAAC (Yanamayo) & 90 & 18 & 20.0 & 4 & 4.4 \\
Chillihua (Chiaraje) & 85 & 1 & 1.2 & 0 & 0 \\
Chillihua (Piti) & 45 & 7 & 15.6 & 0 & 0 \\
Silli & 42 & 20 & 47.6 & 0 & 0 \\
IVITA (La Raya) & 40 & 2 & 5.0 & 5 & 12.5 \\
IVITA (Fundo) & 16 & 1 & 6.3 & 0 & 0 \\
\hline Total & 698 & 239 & 34.2 & 39 & 5.6 \\
\hline
\end{tabular}

Cuadro 3. Relación entre el diagnóstico de Cryptosporidium parvum en alpacas madres y crías en la provincia de Canchis

\begin{tabular}{lcccc}
\hline & \multirow{2}{*}{ Resultado } & \multicolumn{3}{c}{ Presencia de Cryptosporidium spp en la madre } \\
\cline { 3 - 5 } & & Negativo & Positivo & Total \\
\hline Presencia de & Negativo & $442(96 \%)$ & $217(91 \%)$ & $659(94 \%)$ \\
$\begin{array}{l}\text { Cryptosporidium } \\
\text { spp en la cría }\end{array}$ & Positivo & $17(4 \%)$ & $22(9 \%)$ & $39(6 \%)$ \\
\hline & Total & $459(100 \%)$ & $239(100 \%)$ & $698(100 \%)$ \\
\hline
\end{tabular}

Cuadro 4. Análisis de riesgo (Odds Ratio) en relación al diagnóstico de Cryptosporidium parvum en crías de alpaca mediante el modelo de regresión logística

\begin{tabular}{lcccc}
\hline Diag nóstico en la cría & OR & IC menor & IC mayor & Valor de $\mathrm{p}$ \\
\hline Diagnóstico en madres & 2.0981 & 1.0292 & 4.2773 & 0.041 \\
Diarrea en madres & 1.5008 & 0.4233 & 5.3206 & 0.53 \\
Sexo en crías & 0.5608 & 0.2694 & 1.1673 & 0.122 \\
Lugar & 0.229 & 0.0763 & 0.6876 & 0.009 \\
\hline
\end{tabular}


rrea pero positivas a Cryptosporidium. En total, el 6\% (39/698) de las crías fueron positivas (Cuadro 1).

La frecuencia de Cryptosporidium en las madres y crías, según las comunidades, se muestra en el Cuadro 2. La frecuencia de ocurrencia fue muy variable en las madres (desde 0 en UNSAAC-Viscanchi a $74.5 \%$ en Choquecota), mientras que en cuatro comunidades no hubo muestras positivas en crías (Cuadro 2). El sexo sólo se pudo registrar en 573 crías (350 hembras y 223 machos), y el análisis de las muestras demostró que el $6.6 \%$ de las hembras y el $5.8 \%$ de los machos fue positivo a este patógeno.

Al relacionar los diagnósticos de las madres con sus respectivas crías se obtuvo solamente el $9 \%$ de madres positivas con crías positivas (Cuadro 3). Los resultados de la regresión logística para determinar el riesgo de que una madre diagnosticada positiva a Cryptosporidium tenga una cría en semejantes condiciones se observa en el Cuadro 4.

\section{Discusión}

Los trabajos epidemiológicos realizados en la década del 90 determinaron la prevalencia de la criptosporidiosis en alpacas neonatas (López et al., 2009) y el papel de este patógeno como factor de riesgo en la presentación de diarreas en estas crías (López, 1997). Así mismo, los trabajos de Caso-Control confirmaron que el Cryptosporidium es un factor de riesgo para su presentación (Molina et al, 2009; Villacorta et $a l .$, 2009). Sin embargo, queda por determinar las fuentes de infección y su papel como factores de riesgo para la presentación de la criptosporidiosis en alpacas neonatas.

Comprender la epidemiología de la enfermedad era y es crucial para proponer medidas de control adecuadas. En el caso de las alpacas, las fuentes de infección podrían ser otras especies domésticas, roedores, felinos silvestres e incluso el hombre (Fayer y Ungar, 1986; Mtambo et al., 1991). Tampoco se podría descartar que otras alpacas adultas mantuviesen la infección de año a año, como se describió en otras especies domésticas (Ortega-Mora y Wright, 1994).

$\mathrm{Al}$ analizar los datos de las madres y de las crías mediante una regresión logística se obtuvo un OR de 2.098, lo cual permite inferir que una cría con madre positiva a Cryptosporidium spp tiene dos veces más riesgo de infectarse con el parásito que una cría con madre negativa $(\mathrm{p}<0.05)$. Existen dos diseños epidemiológicos para medir factores de riesgo de enfermedad, el de cohortes, que mide directamente el riesgo relativo en función a las incidencias y el de caso control, que no calcula incidencias sino mide en base a la razón de riesgos (Odds ratio) entre poblaciones con y sin el factor de riesgo. En este caso, se empleó el diseño de caso control y se calculó que por cada alpaca con diarrea nacida de madre con diagnóstico negativo a Cryptosporidium habría 2.098 alpacas que tendrían diarrea nacidas de madres con diagnóstico positivo a Cryptosporidium. $\mathrm{La}$ probabilidad de observar esta razón de riesgos debido al azar es menor a 0.05. Esta razón de riesgos para Cryptosporidium y diarrea de alpacas neonatas ha sido descrita con anterioridad para alpacas de Perú (López, 1997).

El presente estudio confirma que uno de los posibles factores de riesgo para la presentación de Cryptosporidium spp en las crías es la presencia de Cryptosporidium spp en la madre. Diversas variables confundentes fueron analizadas como presencia de diarrea en las madres, el sexo de la cría y el lugar de crianza, pero ninguno de ellos afectó el resultado.

Este resultado podría deberse a la permanencia de la madre con la cría. Estudios de criptosporidiosis en bovinos indican que los terneros que permanecen con sus madres por un tiempo mayor a una hora tienen más 
probabilidades de presentar diarrea y mayor cantidad de ooquistes de Cryptosporidium spp que aquellos que fueron separados de sus madres una hora después del parto (TrotzWilliams et al., 2007). Una posibilidad adicional es la falla en la transferencia de anticuerpos calostrales, ya que se ha demostrado que terneros infectados con Cryptosporidium spp eliminan ooquistes cuando las concentraciones séricas de gamaglobulina están bajas, lo que a su vez podría indicar que el parásito es un patógeno oportunista (López et al., 1988).

En la alpaca no ocurre el paso de anticuerpos transplacentarios debido al tipo de placentación epitelio-corial de modo que las inmunoglobulinas maternas se transfieren al neonato por medio del calostro (Steven et al., 1980). Se sabe que la concentración de proteína total en el suero es similar y constante en la alpaca gestante y no gestante (6.9$7.0 \mathrm{~g} / 100 \mathrm{ml}$ ) (Vallenas, 1958), y que en la llama, la concentración de proteína total en el calostro hasta las 96 horas del parto es el doble del contenido en la leche (Ramírez et $a l .$, 1981). Las crías neonatas presentan una baja concentración de proteína sérica $(6.4 \mathrm{~g} /$ $100 \mathrm{ml}$ ), que disminuye durante las 2 primeras semanas de edad $(5.7 \mathrm{~g} / 100 \mathrm{ml})$, para aumentar en forma progresiva con la edad (Ramírez et al., 1981). Garmendia y McGuire (1987) demostraron que las crías nacen virtualmente agamaglobulinémicas con $0.3 \pm 0.1$ $\mathrm{mg} / \mathrm{ml}$, donde la concentración de IgG se incrementa en forma lineal durante las 24 horas después del nacimiento, llegando a 30.0 $\mathrm{mg} / \mathrm{ml}$, y la IgM alcanza un valor de $4.2 \mathrm{mg} / \mathrm{ml}$, para decaer a la mitad de su valor en los días siguientes.

En ovinos, el número de ooquistes eliminado por los adultos es mayor durante las primeras semanas del periodo post-parto, debido a la inmunosupresión que las hace susceptibles a reiniciar el ciclo endógeno del Cryptosporidium, aumentando el riesgo de infección en las crías (Ortega-Mora y Wright, 1994). La diarrea en estos casos puede o no estar presente, como ocurre en las personas
(Carvalho-Almeida et al., 2006), y el incremento de casos puede estar influenciado por el mayor número de nacimientos y el hacinamiento de los animales (Ortega-Mora et al., 1999). Esto se confirmó en el estudio donde la presencia de madres con diarrea no fue un factor de riesgo ni de protección para la presencia de Cryptosporidium spp en las crías. La ocurrencia de diarrea no siempre se asocia con criptosporidiosis en animales adultos y, más bien, la diarrea está asociada a otros agentes tales como parásitos, bacterias, y virus (Ramírez, 1991). No obstante, a pesar que hay infecciones concurrentes con otros enteropatógenos, así como el efecto de los factores ambientales, de manejo y nutricionales, varios autores coinciden en la importancia que tiene el Cryptosporidium spp como patógeno primario causante de diarrea aguda (Moore y Zeman, 1991).

El sexo no fue un factor de riesgo ni de protección para la presentación de Cryptosporidium spp, lo cual corrobora trabajos previos que señalan que la diarrea neonatal no tiene predilección por sexo alguno (Caman, 1996; Tribeño, 1997). El análisis con respecto al lugar mostró que el lugar de crianza es un factor de protección $(p<0.009)$ para las crías pertenecientes a las comunidades en relación a las crías de las granjas de las universidades. Esto es posible debido a que los animales en las granjas experimentales se encuentran usualmente en mayor número por unidad de área de pastura, en tanto que las comunidades poseen grandes extensiones de terreno y, por lo tanto, se reduce el contacto entre los animales, disminuyendo el riesgo de infección (Garber et al., 1994; Mohammed et al., 1999). El hacinamiento ayuda a la diseminación de la enfermedad, ya que las alpacas adultas actuarían como portadores asintomáticos (López, 1997; Ortega-Mora et al., 1999; Acha y Szyfres, 2003).

\section{Conclusiones}

La presencia de Cryptosporidium parvum en la alpaca madre es uno de los 
factores de riesgo para la presentación del patógeno en sus respectivas crías, mas no así la presencia de diarrea en las madres o el sexo de la cría.

\section{Literatura Citada}

1. Acha P, Szyfres B. 2003. Zoonosis y enfermedades transmisibles comunes al hombre y a los animales. Vol III. Parasitosis. $3^{\circ}$ ed. Washington: Organización Panamericana de Salud. 413 p.

2. Angus KW. 1990. Cryptoposridiosis in ruminants. In: Cryptosporidiosis of man and animals. Boca Raton, Florida: CRC Press. p 83-103.

3. Caman V. 1996. Prevalencia de criptosporidiosis en alpacas neonatas en el centro alpaquero de la SAISMaranganí Cuzco. Tesis Bachillerato. Lima: Facultad de Medicina Veterinaria, Univ. Nacional Mayor de San Marcos. $38 \mathrm{p}$.

4. Cármenes P, Rojo-Vázquez FA, Muñoz M, Ortega-Mora LM. 1993. Gastroenteritis infecciosas y parasitarias de los corderos y cabritos. Ovis 27: 7-8.

5. Carvalho-Almeida T, Pinto P, Cuadros $C$, Torres MA, Kanamura $H$, Casimiro, A. 2006. Detection of Cryptosporidium sp. in non diarrheal faeces from children, in a day care center in the city of Sao Paulo, Brazil. Rev Inst Med Trop Sao Paulo 48: 27-32.

6. Casemore R, Armstrong M, Sans $R$. 1985. Laboratory diagnosis of cryptosporidiosis. J Clin Pathol 38: 1337-1341.

7. [CONAM] Consejo Nacional del Ambiente. 1999. Punto focal Cusco: Estrategia regional para la conservación y utilización sostenible de la biodiversidad biológica. Cusco: Universidad Nacional San Antonio de Abad. 66 p.

8. [FAO] Organización de las Naciones Unidas para la Agricultura y la Alimentación. 2005. Situación actual de camélidos sudamericanos en el Perú. Proyecto de cooperación técnica en apoyo a la crianza y aprovechamiento de los camélidos sudamericanos en la región andina. Santiago de Chile: FAO. 63 p.

9. Fayer R, Urgar L. 1986. Cryptosporidium spp. and cryptoposridiosis. Microbiol Rev 50: 458-483.

10. Fayer R, Morgan U, Upton S. 2000. Epidemiology of Cryptosporidium: transmission, detection and identification. Int J Parasitol 30: 1305-1322.

11. Foroca D, Zanabria V, Málaga J, Vilca F. 2001. Cryptosporidiosis en alpacas crías del centro de investigación y producción La Raya-UNA-Puno. Tesis Medico Veterinario. Puno: Universidad Nacional del Altiplano. $48 \mathrm{p}$.

12. Garmendia AE, McGuire TC. 1987. Mechanism and isotypes involved in passive immunoglobulin transfer to the newborn alpaca (Lama pacos). Am J Vet Res 48: 1465-1471.

13. Garber LP, Salman MD, Hurd HS, Keefe T, Schlater JL. 1994. Potential risk factors for Cryptosporidium infection in dairy calves. J Am Vet Med Assoc 2005: 86-91.

14. Henricksen SA, Pohlenz JFL. 1981. Staining of Cryptosporidium by a Modified Ziehl-Neelsen technique. Acta Vet Scand 22: 594-596.

15. López JW, Allen SD, Mitchell J, Quinn M. 1988. Rotavirus and Cryptosporidium shedding in dairy calf feces and its relationship to colostrum immune transfer. J Dairy Sci 71: 1288-1294.

16. López T. 1997. Estudio epidemiológico de la criptosporidiosis en alpacas neonatas. Tesis doctoral. España: Universidad de León. 176 p.

17. López-Urbina MT, González AE, Gomez-Puerta LA, Romero-Arbizu MA, Perales-Camacho RA, Rojo Vázquez FA, et al. 2009. Prevalence of neonatal cryptosporidiosis in Andean alpacas (Vicugna pacos) in Peru. The Open Parasitology Journal 3: 9-13.

18. Mohammed HO, Wade SE, Schaaf S. 1999. Risk factors associated with Cryptoporidium parvum infection in dairy cattle in southeastern New York State. Vet Parasitol 83: 1-13. 
19. Molina D, López MT, González AE, Gómez LA, Pezo SD. 2009. Cryptosporidium parvum como factor de riesgo en la diarrea neonatal en alpacas de Puno. Rev Inv Vet, Perú 20: 263-269.

20. Moore DA, Zeman DH. 1991. Cryptosporidiosis in neonatal calves: 277 cases (1986-1987). JAVMA 198: 1969-1971.

21. Mtambo MMA, Nash AS, Blewett DA. 1991.Cryptosporidium infection in cats: prevalence of infection in domestic and feral cats in the Glasgow area. Vet Rec 129: 502-504.

22. Mũ̃oz FM, Ortega Mora LM, Carmenes DP. 1993. Tratado de patología y producción ovina: gastroenteritis infecciosa y parasitaria de los corderos y cabritos. Ovis 27: 76-86.

23. Ortega-Mora L, Wright S. 1994. Agerelated resistance in ovine Cryptosporidiosis: patterns of infection and humoral immune response. Infec Immun 62:5003-5009.

24. Ortega-Mora LM, Gomez M, RojoVásquez F. 1999. Criptosporidiosis. En: Cordero del Campillo M, Rojo Vasquez FA, Martinez Fernandez AR, Sanchez Acedo MC, Hernandez Rodriguez S, Navarrete Lopez- Cozar I, et al. Parasitología veterinaria. Madrid: GrawHill Interamericana. p 213-221.

25. Ramírez A, Ogi A, Sumar J, Valdivia R. 1981. Características físico-químicas de la leche de llama. Mem IV Conv Inter Camel Sudam. Punta Arenas-Chile. p 20-21.
26. Ramírez A. 1991. Enfermedades Infecciosas. En: Fernández-Baca S (ed). Avances y perspectivas del conocimiento de los camélidos sudamericanos. Santiago: FAO. p 263-323.

27. Rothman K, Greenland S. 1998. Modern epidemiology. $2^{\text {nd }}$ ed. Philadelphia, USA: Lippincott Williams \& Wilkins. $738 \mathrm{p}$.

28. Steven DH, Burton GJ, Sumar J, Nathanielsz PW. 1980. Ultrastructural observations on the placenta of the alpaca (Lama pacos). Placenta 1(1): 21-32.

29. Tribeño D. 1997. Prevalencia de Cryptosporidium parvum en alpacas neonatas de Caylloma-Arequipa. Tesis de Médico Veterinario. Lima: Facultad de Medicina Veterinaria, Univ. Nacional Mayor de San Marcos. 30 p.

30. Trotz-Williams LA, Wayne Martin S, Leslie KE, Duffield T, Nydam DV, Peregrine AS. 2007. Calf-level risk factors for neonatal diarrhea and shedding of Cryptosporidium parvum in Ontario dairy calves. Prev Vet Med 82: 12-28.

31. Vallenas A. 1958. Las proteínas totales y fraccionadas del suero sanguíneo de alpacas. Algunas variaciones fisiológicas. Rev Fac Med Vet, UNMSM 2: 41-49.

32. Villacorta C, López MT, González AE, Gómez LA, Pezo SD. 2009. Evaluación de Cryptosporidium parvum como factor de riesgo para la presentación de diarrea neonatal en alpacas en Cusco. Rev Inv Vet, Perú 20: 277-284. 